\title{
Differential conductance as a promising approach for rapid DNA sequencing with nanopore-embedded electrodes
}

\author{
Yuhui He, ${ }^{1}$ Lubing Shao, ${ }^{1}$ Ralph H. Scheicher, ${ }^{2}$ Anton Grigoriev, ${ }^{2}$ Rajeev Ahuja, ${ }^{2,3}$ \\ Shibing Long, ${ }^{1}$ Zhuoyu Ji, ${ }^{1}$ Zhaoan $\mathrm{Yu},{ }^{1}$ and Ming Liu ${ }^{1, a)}$ \\ ${ }^{1}$ Laboratory of Nano-Fabrication and Novel Devices Integrated Technology, Institute of Microelectronics, \\ Chinese Academy of Sciences, Beijing 100029, People's Republic of China \\ ${ }^{2}$ Department of Physics and Astronomy, Condensed Matter Theory Group, Box 516, Uppsala University, \\ SE-751 20 Uppsala, Sweden \\ ${ }^{3}$ Department of Materials and Engineering, Applied Materials Physics, Royal Institute of Technology \\ (KTH), SE-100 44 Stockholm, Sweden
}

(Received 31 March 2010; accepted 16 April 2010; published online 26 July 2010)

\begin{abstract}
We propose an approach for nanopore-based DNA sequencing using characteristic transverse differential conductance. Molecular dynamics and electron transport simulations show that the transverse differential conductance during the translocation of DNA through the nanopore is distinguishable enough for the detection of the base sequence and can withstand electrical noise caused by DNA structure fluctuation. Our findings demonstrate several advantages of the transverse conductance approach, which may lead to important applications in rapid genome sequencing. (C) 2010 American Institute of Physics. [doi:10.1063/1.3467194]
\end{abstract}

The proposals to electrically sequence DNA during its translocation through a nanopore have become a focus of recent research, due to its prospect of low-cost (less than U.S. \$1000) and fast process (in a matter of hours). ${ }^{1-3}$ The original DNA-nanopore experiments showed that when a single-stranded DNA (ssDNA) molecule is electrophoretically driven through a nanopore, the ionic current passing through the pore is sensitive to the bases in the nanopore, opening a horizon for DNA sequencing. ${ }^{4}$ Since then, numerous experimental and theoretical studies have been performed, with the aim to sequence the translocating polynucleotides by probing various physical properties of the molecules. ${ }^{5-8}$ One idea suggests that the transverse tunneling currents during the DNA translocation might be sufficiently localized to sense and identify a single nucleotide, ${ }^{9}$ and is supported by theoretical calculations. ${ }^{10,11}$ This idea is now being actively tested by using the conducting tip of a scanning tunneling microscope over immobilized short polynucleotides on a substrate. ${ }^{12,13}$ However, further calculations showed that structural fluctuations of DNA during the sequencing, which causes a serious deterioration of signal-tonoise ratio, substantially reduce the selectivity and thus remains one of the biggest obstacles for the feasibility of implementing electrical sequencing approaches. ${ }^{14,15}$

Here, we propose and theoretically demonstrate a sequencing approach based on characteristic transverse differential conductance of DNA. The idea is that the four nucle- otides occurring in DNA possess different local electronic densities of states (LDOSs) owing to their distinct chemical structures, and this specificity of different nucleotides can be measured and demonstrated directly by transverse differential conductance curves. From our molecular dynamics (MD) simulations and real-time calculations of LDOS and electrical properties, we predict that those characteristic resonance levels of different nucleotides remain distinguishable even when taking structural fluctuations into account, making transverse differential conductance curves a promising candidate for robust nanopore-based DNA sequencing.

Figure 1(a) shows the working principle of a nanoporebased electrical sequencing setup: ssDNA is driven through the nanopore by a longitudinal electrical field, while transverse tunneling conductance is recorded for the purpose of sequencing. Figures 1(b) and 1(c) present the cross-section of a bare nanopore and a snapshot of translocating ssDNA in $\mathrm{KCl}$ solution. The nanopore is built within a $\mathrm{SiN}-\mathrm{Au}-\mathrm{SiN}$ sandwich structure: $1 \mathrm{~nm}$ thick $\mathrm{Au}$ in the inner layer functions as transverse electrodes, while two 4-6 nm SiN layers at both ends insulate the electrodes from the solution environment. Here four electrodes are defined to provide more flexibility for transverse conductance measurement. The inner diameter of the nanopore is $13 \AA$, sufficiently wide for a ssDNA molecule to pass through, while also narrow enough to achieve a measurable transverse tunneling conductance.
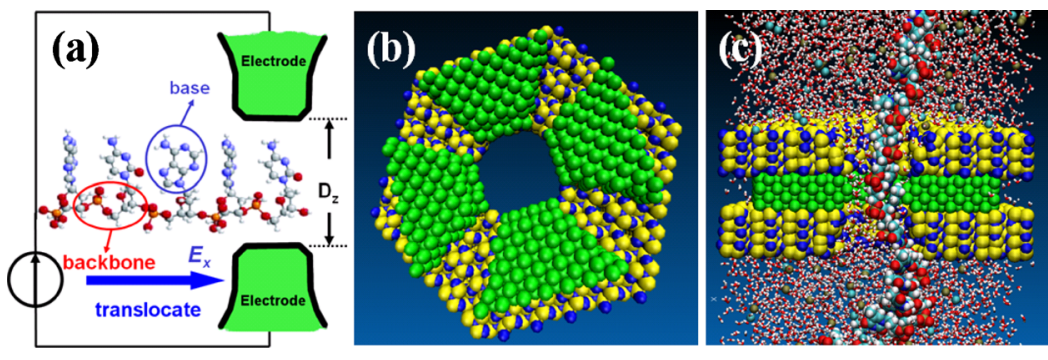

FIG. 1. (Color online) (a) Schematic view of a singlestrand DNA translocating through a nanopore under longitudinal electrical field $\mathrm{E}_{x}$, while transverse tunneling currents are recorded for the purpose of sequencing. (b) Cross-section view of a bare nanopore. (c) Snapshot of nanopore-based DNA sequencing device at working state.

${ }^{a)}$ Electronic mail: liuming@ime.ac.cn. 


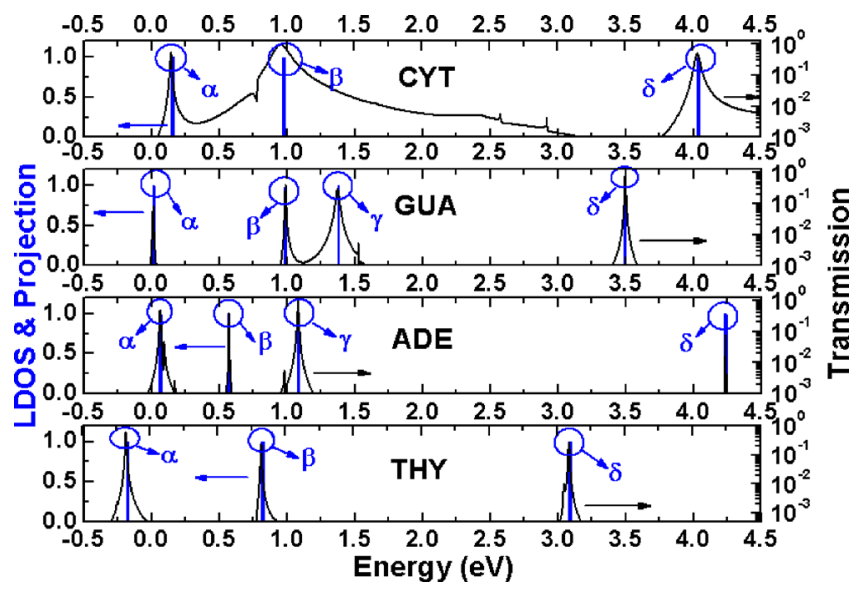

FIG. 2. (Color online) LDOS, their projections on base atoms, and transverse transmission spectra of poly $(\mathrm{dX})_{30}(\mathrm{X}=\mathrm{A}, \mathrm{T}, \mathrm{C}, \mathrm{G})$ at some typical snapshots during the translocation. Here, the Fermi level is calibrated to that of bulk gold. Several characteristic resonance levels of nucleotides are marked.

The simulation is implemented in the following way: first, the translocation of short homogeneous polynucleotides $\left[\operatorname{poly}(\mathrm{dX})_{30}\right.$, with $\left.\mathrm{X}=\mathrm{A}, \mathrm{T}, \mathrm{C}, \mathrm{G}\right]$ through the nanopore is simulated with NAMD, ${ }^{16}$ a classical molecular dynamics package using Amber force-field parameters. ${ }^{17,18}$ Then the real-time atomic configuration is extracted from MD trajectory files, and the corresponding real-time electronic structure of DNA during the translocation process is calculated within the extended Hückel model using YAEHMOP. ${ }^{19}$ Finally, characteristic energy levels are picked out for each kind of nucleotide, and the corresponding transverse conductance $G\left(V_{0}\right)$ is calculated using the Landauer-Büttiker formalism and nonequilibrium Green's function technique. ${ }^{20}$

Figure 2 plots the transmission spectra in the transverse directions, nucleotide DOSs and their projections on base atoms at some typical moment during the translocation. This figure reveals a great deal of useful information and motivates the proposal for our sequencing approach: first, the DOS near the Fermi level originates from base atoms, not backbone atoms, ${ }^{21}$ and thus can serve for the purpose of sequencing; second, the resonance transmission observed in this figure indicates that the transverse electrical properties are determined by resonance levels of nucleotides; and third, each nucleotide has its characteristic resonance levels, making unique transmission spectra possible for each base type.

Let us first review the well-known formula for the transverse electrical current between electrodes $i$ and $j$, as follows:

$$
I_{i j}(V)=\frac{2 e}{h} \int d \epsilon T_{i j}(\epsilon)\left[f\left(\epsilon-\mu_{i}\right)-f\left(\epsilon-\mu_{j}\right)\right],
$$

where $V=\mu_{i}-\mu_{j}$ is the bias voltage, $T_{i j}$ is the transmission coefficient, and $f(\epsilon-\mu)$ is the Fermi distribution function. Equation (1) shows that the transverse electrical current is proportional to the sum of those transmission peaks that lie inside the bias voltage window. Now, calculating the derivative $d I / d V$, we can obtain the differential conductance as follows:

$$
G_{i j}\left(\mu_{F}\right)=\frac{e^{2}}{h k_{B} T} \int d \epsilon T_{i j}(\epsilon)\left[2+\cosh \frac{\epsilon-e \mu_{F}}{k_{B} T}\right]^{-1},
$$

where $G_{i j}$ is the zero-bias differential conductance be-
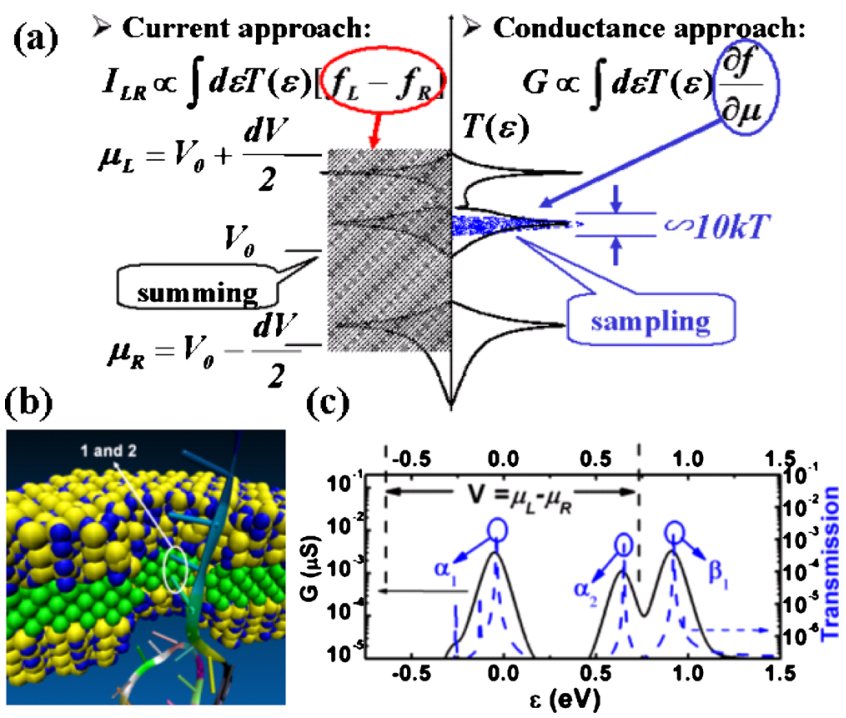

FIG. 3. (Color online) (a) Comparison between electrical current approach and differential conductance approach: $I$ is proportional to the sum of transmissions within the voltage bias window, while $G$ corresponds to the sampling of transmission probability at the Fermi level $\mu_{0}$. (b) Translocation of poly $(\mathrm{dT})_{30}$ at the moment when two neighboring nucleotides (1 and 2) are in the vicinity of the electrodes, contributing both to the conductance. (c) Snapshot of conductance and transmission spectra for poly $(\mathrm{dT})_{30}$ translocating through nanopore.

tween electrodes $i$ and $j, \mu_{F}$ is the Fermi level, and $T$ is the temperature. Owing to its sampling nature [the term in square brackets of Eq. (2)], transverse differential conductance spectra can reveal directly the characteristic transmission features at the Fermi energy. This indicates a potential sequencing approach for the nanopore-embedded electrodes setup: we can utilize transverse differential conductance to characterize electronic signatures of each nucleotide.

It is of interest to make a comparison between this differential conductance approach and the electrical current approach. The difference between the summing nature of the electrical current approach and the sampling nature of differential conductance approach is demonstrated in Fig. 3(a). Considering the possibility that several nucleotides inside the nanopore contribute to the transverse conduction simultaneously, due to insufficiently thin nanoelectrodes, the summing of those transmission peaks caused by different nucleotides could entail misleading results, i.e., data from the electrical current approach would become invalid for sequencing if more than 1 nucleotide are simultaneously close enough to electrodes and contribute to the conductance. Figure 3(b) gives such an example: at some moment during a poly $(\mathrm{dT})_{30}$ translocating through a nanopore, two neighboring nucleotides, marked by 1 and 2, are close enough to the electrodes and thus contribute to the conductance simultaneously. Accordingly, there are two neighboring peaks $\alpha_{1}$ and $\alpha_{2}$ inside the transverse bias window $V=\mu_{L}-\mu_{R}$ on the transverse transmission spectrum, as seen in Fig. 3(c). By projecting the resonance levels onto atomic orbits, we find that $\alpha_{1}$ and $\alpha_{2}$ originate from nucleotide 1 and 2 separately. In this case, the transverse electrical current $I$ is the summing of these two transmission peaks and does not achieve singlenucleotide resolution. On the other side, the sampling nature of differential conductance makes no more than 1 transmission peak selected each time, and thus circumvents those inevitable miscountings in the electrical current approach. 


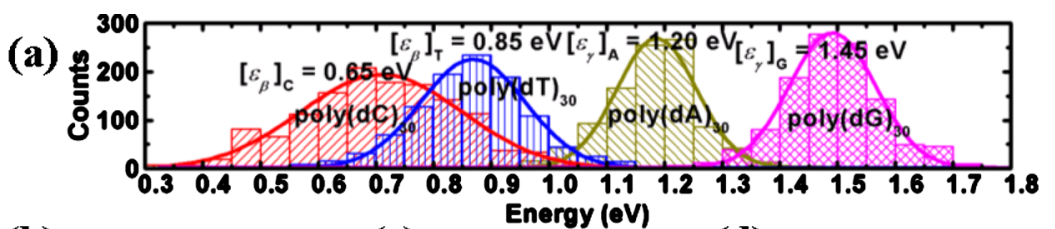

(b)

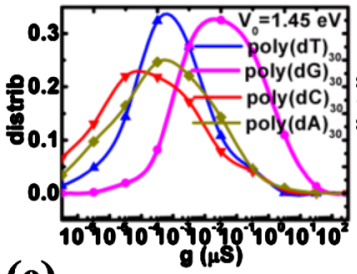

(c)

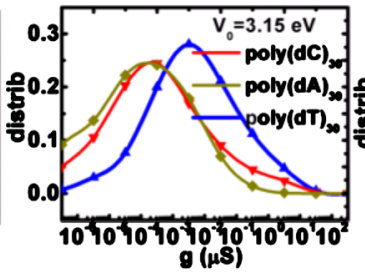

(d)

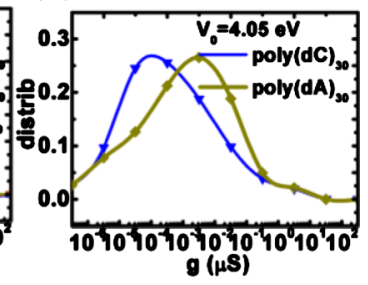

(e)

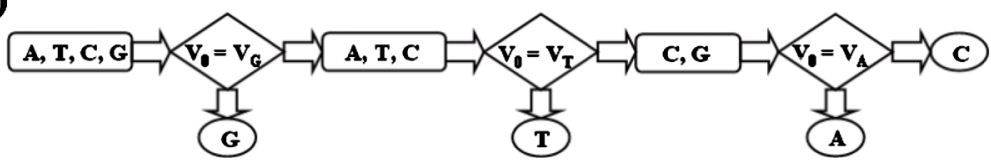

FIG. 4. (Color online) (a) Distributions of resonance levels of poly $(\mathrm{dX})_{30}(\mathrm{X}=\mathrm{A}, \mathrm{T}, \mathrm{C}, \mathrm{G})$ during the translocation. (b) Setting $V_{0}$ at $\epsilon_{\gamma}$ of guanine $(1.45 \mathrm{eV})$, and the conductance distribution $G\left(V_{0}\right)$ of guanine differs from that of other three nucleotides by two to three orders. (c) Setting $V_{0}$ at $\epsilon_{\delta}$ of thymine $(3.15 \mathrm{eV})$, thymine is identified from cytosine and adenine. (d) Setting $\epsilon_{\delta}$ of adenine $(4.05 \mathrm{eV})$ and the remaining two nucleotides are distinguished from each other. (e) The sequencing flowchart.
The question of whether the fluctuation of characteristic resonance levels during the translocation would destroy the distinction of LDOS between different nucleotides can be answered in Fig. 4(a), where the distributions of characteristic resonance levels of four kinds of nucleotides are plotted. It is clearly seen that the characteristic resonance levels of the different nucleotides remain distinguishable taking realtime fluctuations into account. Therefore, this can be regarded as a convincing proof that the transverse differential conductance approach is able to withstand DNA structure fluctuations during the sequencing process and thus is an efficacious sequencing approach.

Figures 4(b)-4(e) show a flowchart representation of the sequencing approach: at one characteristic resonance level of guanine $\left(V_{0}=V_{G} \approx 1.45 \mathrm{eV}\right)$, real-time transverse conductance of poly $(\mathrm{dG})_{30}$ is two to three orders larger than that of the other three homogeneous polynucleotides, leading to the identification of guanine as seen in Fig. 4(b). In the same way, thymine, adenine and cytosine are determined step by step at their characteristic energies in transverse conductance curves, as plotted in Figs. 4(c) and 4(d).

In summary then, we have proposed and theoretically justified a sequencing protocol based on the characteristic transverse differential conductance of ssDNA during its translocation through a nanopore. The simulations show that transverse conductance curves determined by the characteristic resonance levels of each nucleotide are distinguishable enough for the sequencing of DNA, and are sufficiently robust to withstand DNA structure fluctuations during the sequencing process. In practice, moving molecular orbitals have never been identified yet with $d I / d V$ spectroscopy, however the inverse problem, when the gold tip is moving along the molecule, was recently solved. ${ }^{22}$ Our theoretical results can provide a guide for future realizations of inexpensive and rapid whole-genome sequencing applications. Finally, we would also like to draw attention to a very recently proposed hypothetical setup for DNA sequencing which does not utilize a nanopore, but instead relies on a graphene nanogap, which simultaneously fulfills the role of separating membrane and single-atom thick electrodes. ${ }^{23}$ It is particularly noteworthy that in that work too, the differential conductance was considered in the form of the first derivative $d I / d V$ and the third derivative $d^{3} I / d V^{3}$ to uniquely identify the four nucleobase types.

We gratefully acknowledge financial support from the National Science Fund for Distinguished Young Scholars (Grant No. 60825403), China Ministry of Science and Technology (Contract No. 2010CB934200), the Swedish Foundation for International Cooperation in Research and Higher Education (STINT), the Swedish Research Council (VR), Wenner-Gren Foundations, Carl Tryggers Stiftelse för Vetenskaplig Forskning, and the Uppsala University UniMolecular Electronics Center ( $\left.\mathrm{U}^{3} \mathrm{MEC}\right)$.

${ }^{1}$ D. Branton, et al., Nat. Biotechnol. 26, 1146 (2008).

${ }^{2}$ M. Zwolak and M. Di Ventra, Rev. Mod. Phys. 80, 141 (2008).

${ }^{3}$ C. Dekker, Nat. Nanotechnol. 2, 209 (2007).

${ }^{4}$ J. J. Kasianowicz, E. Brandin, D. Branton, and D. W. Deamer, Proc. Natl. Acad. Sci. U.S.A. 93, 13770 (1996).

${ }^{5}$ G. Sigalov, et al., Nano Lett. 8, 56 (2008).

${ }^{6}$ H. He, R. H. Scheicher, R. Pandey, A. R. Rocha, S. Sanvito, A. Grigoriev, R. Ahuja, and S. P. Karna, J. Phys. Chem. C 112, 3456 (2008).

${ }^{7}$ S. Chang, et al., Nat. Nanotechnol. 4, 297 (2009).

${ }^{8}$ S. Chang, et al., Nano Lett. 10, 1070 (2010).

${ }^{9}$ J. W. Lee and T. Thundat, DNA and RNA Sequencing by Nanoscale Reading Through Programmable Electrophoresis and Nanoelectrode-Gated Tunneling and Dielectric Detection, U.S. Patent No. 6, 905, 586, June 14 (2005).

${ }^{10}$ M. Zwolak and M. Di Ventra, Nano Lett. 5, 421 (2005).

${ }^{11}$ J. Lagerqvist, M. Zwolak, and M. Di Ventra, Nano Lett. 6, 779 (2006).

${ }^{12}$ M. Xu, R. G. Endres, and Y. Arakawa, Small 3, 1539 (2007).

${ }^{13}$ E. Shapir, et al., Nature Mater. 7, 68 (2008).

${ }^{14}$ J. Lagerqvist, M. Zwolak, and M. Di Ventra, Biophys. J. 93, 2384 (2007).

${ }^{15}$ R. Zikic, et al., Phys. Rev. E 74, 011919 (2006).

${ }^{16}$ L. Kale, J. Comput. Phys. 151, 283 (1999).

${ }^{17}$ W. Cornell, et al., J. Am. Chem. Soc. 117, 5179 (1995).

${ }^{18} \mathrm{We}$ have used the program www.ks.uiuc.edu/Training/tutorials to generate our codes. A reference is given for the program: J. Comer, V. Dimitrov, Q. Zhao, G. Timp, and A. Aksimentiev, Biophys. J. 96, 593 (2009).

${ }^{19}$ G. Landrum, Ph.D. dissertation, Cornell University, 1997.

${ }^{20} \mathrm{M}$. Di Ventra, Electrical Transport in Nanoscale Systems (Cambridge University Press, Cambridge, 2008).

${ }^{21}$ Nucleotide DOS was calculated in the extended Hückel model using the YAEHMOP program, which yields results in good agreement with densityfunctional approach.

${ }^{22}$ W.-H. Soe, et al., Phys. Rev. Lett. 102, 176102 (2009).

${ }^{23}$ H. W. Ch. Postma, Nano Lett. 10, 420 (2010). 